\title{
Temperature (Over)Compensation in an Oscillatory Surface Reaction
}

\author{
Raphael Nagao, ${ }^{\dagger}$ Irving R. Epstein, Ernesto R. Gonzalez, ${ }^{\dagger}$ and Hamilton Varela ${ }^{*}, \dagger$ \\ Instituto de Química de São Carlos, Universidade de São Paulo, C.P. 780, CEP 13560-970, São Carlos - SP, \\ Brasil, and Department of Chemistry and Volen Center for Complex Systems, MS 015, Brandeis University, \\ Waltham, Massachusetts 02454-9110
}

Received: February 15, 2008

\begin{abstract}
Biological rhythms are regulated by homeostatic mechanisms that assure that physiological clocks function reliably independent of temperature changes in the environment. Temperature compensation, the independence of the oscillatory period on temperature, is known to play a central role in many biological rhythms, but it is rather rare in chemical oscillators. We study the influence of temperature on the oscillatory dynamics during the catalytic oxidation of formic acid on a polycrystalline platinum electrode. The experiments are performed at five temperatures from 5 to $25^{\circ} \mathrm{C}$, and the oscillations are studied under galvanostatic control. Under oscillatory conditions, only non-Arrhenius behavior is observed. Overcompensation with temperature coefficient $\left(q_{10}\right.$, defined as the ratio between the rate constants at temperature $T+10{ }^{\circ} \mathrm{C}$ and at $\left.T\right)<1$ is found in most cases, except that temperature compensation with $q_{10} \approx 1$ predominates at high applied currents. The behavior of the period and the amplitude result from a complex interplay between temperature and applied current or, equivalently, the distance from thermodynamic equilibrium. High, positive apparent activation energies were obtained under voltammetric, nonoscillatory conditions, which implies that the non-Arrhenius behavior observed under oscillatory conditions results from the interplay among reaction steps rather than from a weak temperature dependence of the individual steps.
\end{abstract}

\section{Introduction}

Temperature plays a key role in chemical reactions. The dependence of reaction rates on temperature can be expressed as the temperature coefficient, $Q_{10},{ }^{1,2}$ which is defined as the ratio between the reaction rate constant $k$ at a temperature $T+$ $10{ }^{\circ} \mathrm{C}$ and the one at $T$.

$$
Q_{10}=\frac{k_{T+10{ }^{\circ} \mathrm{C}}}{k_{T}}
$$

For an arbitrary temperature interval, we can write

$$
q_{10}=\left(\frac{k_{T_{2}}}{k_{T_{1}}}\right)^{10 /\left(T_{2}-T_{1}\right)}
$$

where $q_{10}$ is the mean temperature coefficient $Q_{10}$ in the temperature interval between $T_{1}$ and $T_{2}$. The Arrhenius activation energy can be written in terms of the mean temperature coefficient,

$$
E_{\mathrm{a}}=R\left(\frac{T_{1} T_{2}}{10}\right) \ln q_{10}
$$

where $R$ is the gas constant.

As a rule of thumb, most chemical and biochemical reactions have temperature coefficients from 2 to $4 .{ }^{1}$ In spite of this strong dependence of reaction rates on temperature, a system such as a network of biochemical reactions might be independent of temperature over a considerable temperature range, which means a $q_{10}$, or $Q_{10}$, value around 1 . This approximate constancy of the oscillatory period is called temperature compensation. ${ }^{3}$ Biological rhythms ${ }^{4}$ are regulated by homeostatic mechanisms

\footnotetext{
* Corresponding author. E-mail: varela@iqsc.usp.br.

$\dagger$ Universidade de São Paulo.

$\ddagger$ Brandeis University.
}

that assure the reliability of the period length or, more generally, the physiological clock functions, independently of temperature changes in the environment. Indeed, temperature compensation is often cited as an essential property of circadian and ultradian rhythms. ${ }^{5-10}$ Cases where the temperature coefficient is considerably smaller than 1 are referred to as overcompensated and reflect the counterintuitive situation in which the process becomes slower as the temperature increases. Such behavior would result, as seen in eq 3 , in a negative activation energy; whereas when $q_{10} \sim 1$, the activation energy approaches zero. Elementary reactions displaying unusual values of the activation energy have been discussed by Benson and co-workers. ${ }^{11-13}$

The concept of antagonistic balance ${ }^{14-16}$ states that for a given set of activation energies, the positive feedback loops can be counterbalanced by negative feedback loops in such a way that temperature compensation is achieved. In this way, homeostasis of the oscillation period would result from the antagonistic balance of all the physicochemical or physiological parameters that influence the component processes of the clock mechanism. ${ }^{16}$ As an example, Huang et al. ${ }^{17}$ reported that temperature compensation of the circadian rhythm in Drosophila could in principle be caused by antagonistic protein-protein interactions composed of inter- and intramolecular steps with similar temperature coefficients. From the biochemical mechanism perspective, it has been suggested that the enzymatic activity can keep its optimum level over a considerable range of temperature and hydrostatic pressure, mainly by tuning the ligand-binding enthalpy and volume. ${ }^{18}$

Chemical systems far from thermodynamic equilibrium have frequently been used as biomimetic models in the investigation of complex dynamics in biological processes. ${ }^{19}$ Classic examples are the electrooxidation of metals as a model of nerve pulse propagation, ${ }^{20,21}$ and the mercury beating heart. ${ }^{22,23}$ In the present context, Ruoff ${ }^{15}$ has suggested that any chemical oscillator can, 
in principle, exhibit temperature compensation. The most wellstudied chemical oscillator, the celebrated Belousov-Zhabotinsky (BZ) reaction, ${ }^{24,25}$ is known to follow the Arrhenius equation to a considerable extent, and a temperature coefficient of 2.5, corresponding to an overall activation energy of $73 \mathrm{~kJ} \mathrm{~mol}^{-1}$, has been found. ${ }^{26-28}$ In addition, this activation energy was determined to be independent of the catalyst. ${ }^{26}$ Using the Oregonator model, Field and Noyes attributed the absence of temperature compensation in the $\mathrm{BZ}$ system to the predominance of a reaction step whose increasing rate constant results in a decrease in the oscillation period. Activation energies of about 58,68 and $89 \mathrm{~kJ} \mathrm{~mol}^{-1}$ have been reported for the BriggsRauscher reaction with benzylmalonic acid, ${ }^{29}$ malonic acid ${ }^{30}$ or 1,4-cyclohexanedione, ${ }^{31}$ respectively, as substrate. Using cyclohexanedione as the organic substrate in the Briggs-Raucher reaction in batch, Kereszturi and Szalai ${ }^{31}$ reported an oscillatory region in which the period is nearly independent of temperature. However, both the transient nature of the oscillations and the limited temperature range $\left(20,25\right.$ and $\left.30^{\circ} \mathrm{C}\right)$ point to the need for more accurate investigations of this system, preferably under flow conditions.

Rábai and co-workers observed temperature compensation in $\mathrm{pH}$ oscillators ${ }^{32}$ and discussed the mechanism underlying the observed phenomena in terms of antagonistic balance. ${ }^{33}$ The effect of temperature on the oscillatory decomposition of hydrogen peroxide in an acidic aqueous solution in the presence of potassium iodate (Bray reaction) has been studied by Kovács et al. $^{34}$ under both batch and flow conditions. Temperature coefficients were found to depend strongly on the concentrations of several reactants, and values from 0.67 to 1.80 were observed in a temperature interval of $10{ }^{\circ} \mathrm{C}$.

We report in this work the experimental observation of strongly non-Arrhenius behavior, namely temperature overcompensation and compensation, in the oscillatory oxidation of formic acid on a polycrystalline platinum electrode in sulfuric acid aqueous media. Oscillations were studied under galvanostatic control at five temperatures in the range between 5 and $25^{\circ} \mathrm{C}$. The results are discussed in terms of the intricate interplay between temperature and the distance from equilibrium in determining the oscillation period and amplitude. Finally, some mechanistic considerations concerning the non-Arrhenius behavior are presented in connection with the state-of-the-art understanding of the electrocatalytic aspects of the oxidation of formic acid on platinum.

\section{Experimental Section}

The working electrode (WE) was smooth polycrystalline platinum with $0.2 \mathrm{~cm}^{2}$ of geometric area and roughness (i.e., the ratio between the real, as measured by the hydrogen adsorption charge, and the geometric area) of about 3.15. The counter electrode (CE) was a net of platinized platinum with a much larger area than that of the WE. A reversible hydrogen electrode (RHE) prepared with the same concentration of sulfuric acid as that of the electrolyte was used as a reference electrode (RE), and all potentials are quoted with respect to it. Before each experiment the WE was cycled at $100 \mathrm{mV} \mathrm{s}^{-1}$ between 0 and $1300 \mathrm{mV}$ vs RHE for about $1 \mathrm{~h}$ to assure a reproducible surface structure. ${ }^{35,36}$ Experiments were carried out with the RE kept in a compartment external to the main cell cylinder at $25^{\circ} \mathrm{C}$, whereas the temperature of the compartment containing the WE was thermostated at different temperatures between 5 and $25^{\circ} \mathrm{C}$. The solutions were prepared with high purity water (Millipore Milli-Q system, $18 \mathrm{M} \Omega \mathrm{cm}$ ), sulfuric acid (Merck, 98\%), and formic acid (Fluka, 98\%), and purged

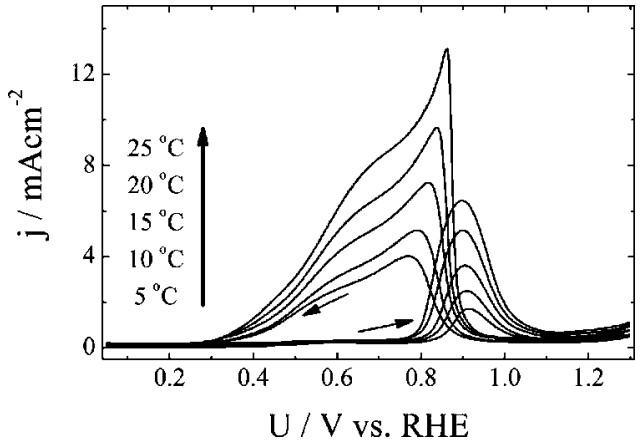

Figure 1. Cyclic voltammograms of the electrooxidation of formic acid on platinum at $5 \mathrm{mV} \mathrm{s}^{-1}$ at several temperatures. Electrolyte: aqueous solution containing $0.60 \mathrm{M} \mathrm{HCOOH}$ and $0.10 \mathrm{M} \mathrm{H}_{2} \mathrm{SO}_{4}$.

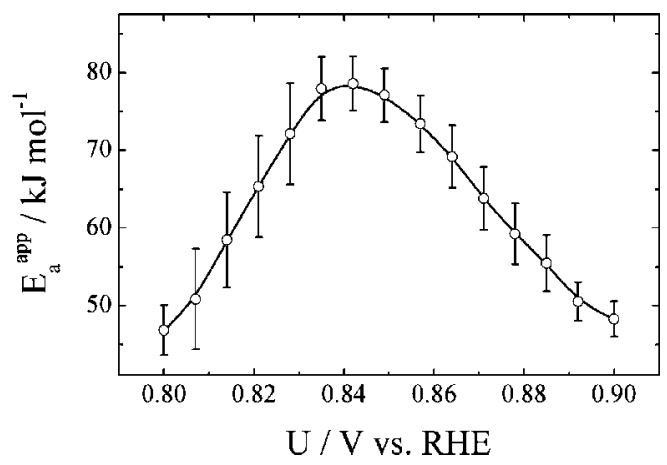

Figure 2. Apparent activation energy at different potentials, as calculated from the current values obtained during the forward sweep of the cyclic voltammograms shown in Figure 1.

with nitrogen for at least $30 \mathrm{~min}$ prior to and during all experiments. The electrochemical measurements were performed with a potentiostat/galvanostat (Autolab/Eco-Chemie, PGSTST30) and the temperature was kept constant via a Marconi thermostat model MA- 184 .

\section{Results}

Figure 1 shows the voltammetric profiles obtained at $5 \mathrm{mV}$ $\mathrm{s}^{-1}$ during the electrooxidation of formic acid on platinum in sulfuric acid media at different temperatures. Overall, the key features such as the presence of a main oxidation wave in the forward sweep in the region between 800 and $1000 \mathrm{mV}$ vs RHE, and the re-activation region in the reverse scan starting at about $900 \mathrm{mV}$ vs RHE are discernible and are in agreement with previously published data. ${ }^{37}$ The main effects of increasing temperature are the increase of the voltammetric currents and a slight decrease in the peak potential separation between the forward and reverse scans.

The temperature effect on the voltammetric profiles given in Figure 1 can be described in terms of the apparent activation energies, $\mathrm{E}_{\mathrm{a}}$ app, calculated via conventional Arrhenius plots of $\ln j$ vs $1 / T$. In this way, the apparent activation energy reflects the sum of all contributions for the electrooxidation reaction at a given potential. Focusing on the potential window between 800 and $900 \mathrm{mV}$ along the forward sweep, the estimated $E_{\mathrm{a}}$ app values are shown in Figure 2. The apparent activation energy is first found to increase with potential from about $47 \mathrm{~kJ} \mathrm{~mol}^{-1}$ at $800 \mathrm{mV}$ to a maximum value of $79 \mathrm{~kJ} \mathrm{~mol}^{-1}$ at $840 \mathrm{mV}$ vs RHE. For higher potentials, $E_{\mathrm{a}}$ app decreases with potential and reaches $48 \mathrm{~kJ} \mathrm{~mol}^{-1}$ at $900 \mathrm{mV}$. The potential interval of 800-900 $\mathrm{mV}$ vs RHE was chosen because it corresponds to the early stages of formic acid oxidation before the current maximum, under voltammetric conditions, cf. Figure 1. Experi- 


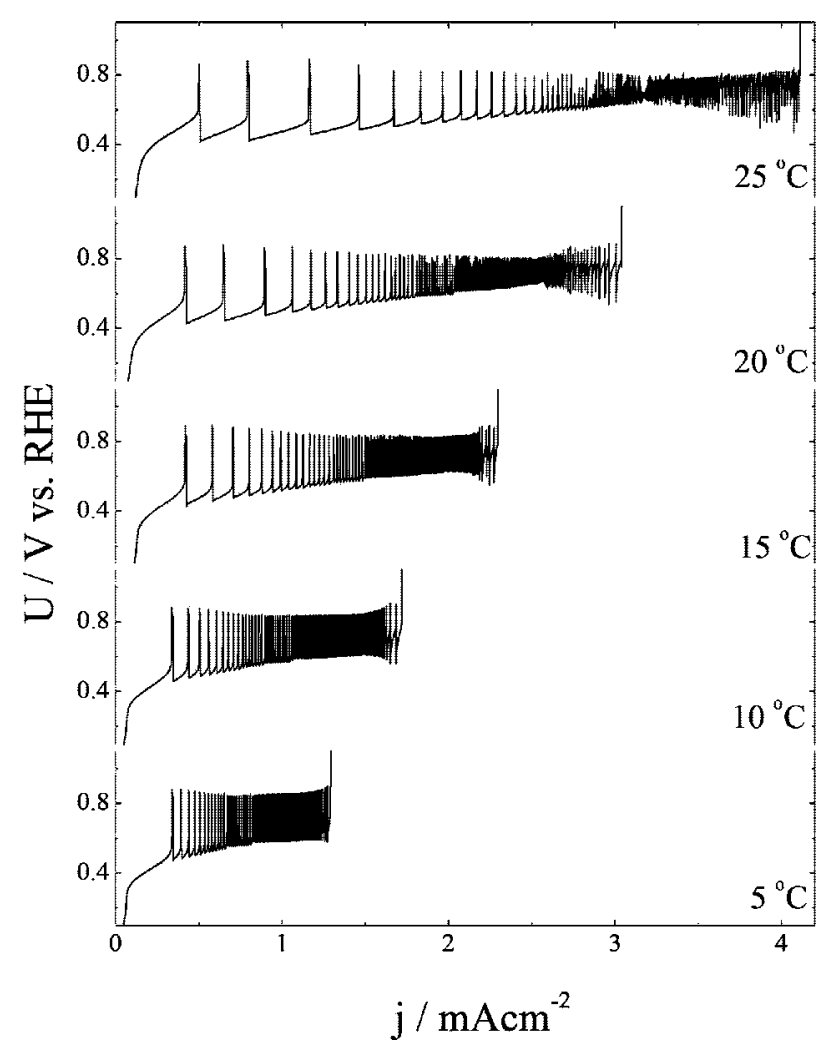

Figure 3. Potential oscillations during the electrooxidation of formic acid on platinum under a galvanodynamic sweep $\left(\mathrm{d} I / \mathrm{d} t=5 \mu \mathrm{A} \mathrm{s}^{-1}\right)$ at different temperatures. Electrolyte: aqueous solution with $0.60 \mathrm{M}$ $\mathrm{HCOOH}$ and $0.10 \mathrm{M} \mathrm{H}_{2} \mathrm{SO}_{4}$.

ments at higher temperatures (not shown) resulted in an apparent activation of about $32 \mathrm{~kJ} \mathrm{~mol}^{-1}$ in the potential range between 850 and $900 \mathrm{mV}$.

Oscillatory kinetics in the electrooxidation of formic acid can be observed under both galvanostatic and potentiostatic control modes, provided that, in the second case, a sufficient resistance exists between the working electrode and the potentiostat. The oscillations discussed here were obtained under galvanostatic control. In this case, the applied current reflects the electron flow through the solid/liquid interface and is a measure of the departure from equilibrium in the system, similarly to the potential difference across the solid/liquid interface under potentiostatic control. ${ }^{38,39}$ A general overview of the oscillatory dynamics is given in Figure 3 in terms of the potential oscillations obtained during a galvanodynamic sweep $(\mathrm{d} I / \mathrm{d} t=$ $5 \mu \mathrm{A} \mathrm{s}^{-1}$ ) at different temperatures. Potential oscillations are born at low current values via a subcritical Hopf bifurcation and disappear at high current values through a saddle-loop bifurcation, where the limit cycle collides with the fixed point in the oxygen evolution branch, similar to the behavior observed for related systems. ${ }^{40,41}$ Already evident in this figure is the trend that the higher the temperature the lower the oscillation frequency. However, the nonstationary nature of the experiments does not allow a rigorous analysis, so discussion of this aspect will be postponed. An important point to be noted in Figure 3 is the dependence of the size of the oscillatory region, $S_{\mathrm{osc}}$, on temperature. A nearly linear increase of $S_{\text {osc }}$ with temperature is observed in this set of data.

Comparison of the oscillation dynamics under stationary conditions (i.e., fixed current) at different temperatures requires consideration of the change of $S_{\text {osc }}$ with temperature. To account for this effect, the applied current was normalized according to the following equation:

$$
I_{\mathrm{N}}^{T}=\frac{j_{\mathrm{osc}}^{T}-j_{\mathrm{osc}, \mathrm{i}}^{T}}{j_{\mathrm{osc}, \mathrm{f}}^{T}-j_{\mathrm{osc}, \mathrm{i}}^{T}}
$$

where $j_{\text {osc }}^{T}$ is the applied current density, and $j_{\text {osc, } i}^{T}$ and $j_{\text {osc }, \mathrm{f}}^{T}$ are the initial and final currents that define the oscillatory potential region, respectively. $j_{\text {osc, } i}^{T}$ and $j_{\text {osc, f }}^{T}$ are estimated from the galvanodynamic sweep. This normalization procedure allows comparison of potential time series obtained at different temperatures for a given applied current. Typical oscillations for $I_{\mathrm{N}}^{T}=0.33$ are given in Figure 4 .

Overall, the waveform is nearly independent of temperature and, besides a small change in its amplitude (vide infra), the main point to be stressed is that the oscillations become slower for increasing temperatures. Indeed, periods of 95 and $5 \mathrm{~s}$ were observed at 25 and $5{ }^{\circ} \mathrm{C}$, respectively. For the time window shown, the number of potential oscillations is $48,19,11,8$, and 3 at $5,10,15,20$, and $25^{\circ} \mathrm{C}$, respectively.

Another set of time series obtained at $I_{\mathrm{N}}^{T}=0.83$ is depicted in Figure 5. As in Figure 4, the general shape of the oscillations is quite similar and nearly insensitive to temperature at this current. However, the oscillations are less steep and faster in this case. Remarkably, in this set the oscillation period depends only weakly on temperature, with the period ranging from 0.87 to $1.36 \mathrm{~s}$ for the different temperatures. The number of potential oscillations for the time window displayed is around 10 (actually $10,10,12,10$ and 8, for increasing temperatures).

An important difference in the waveforms shown in Figures 4 and 5 is that at higher currents (Figure 5) both the active/ passive and the passive/active transitions are less steep, which results in more discernible separation between these transitions in the series depicted in Figure 5. Overall, the oscillation shape is very similar for a given normalized current and different temperatures, which gives support to the validity of the normalization procedure adopted. Changes in the morphology

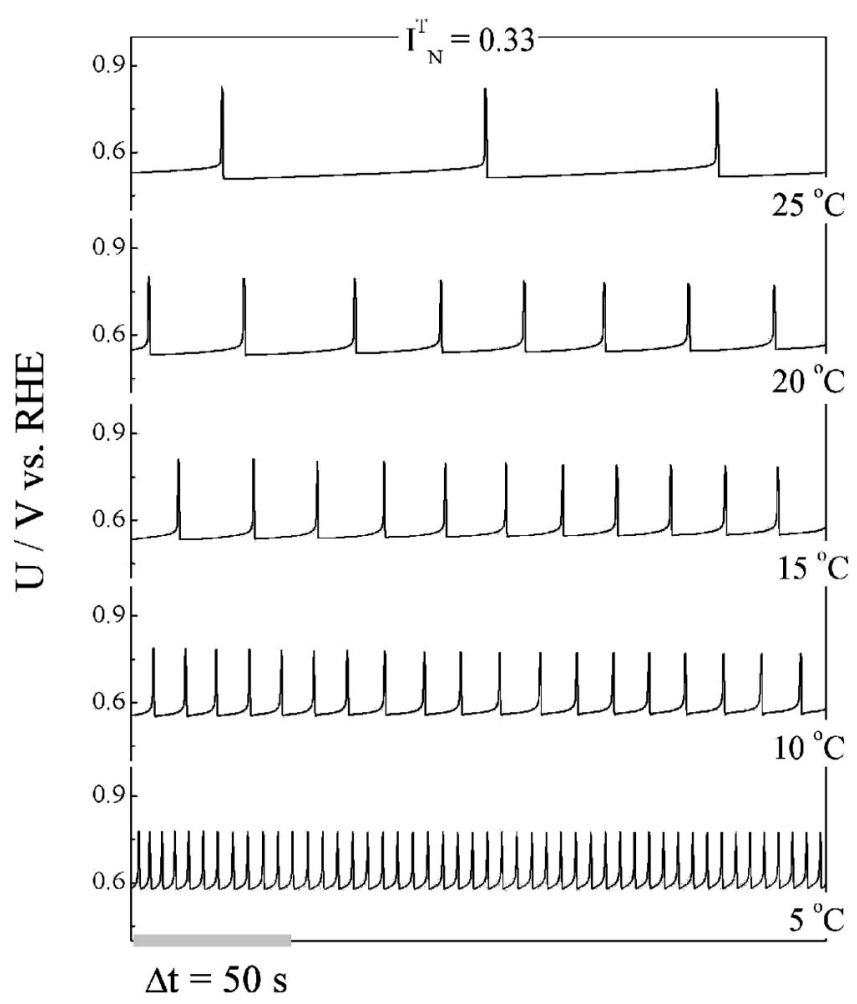

Figure 4. Potential time series at different temperatures and $I_{\mathrm{N}}^{T}=0.33$. Electrolyte: aqueous solution with $0.60 \mathrm{M} \mathrm{HCOOH}$ and $0.10 \mathrm{M} \mathrm{H}_{2} \mathrm{SO}_{4}$. 


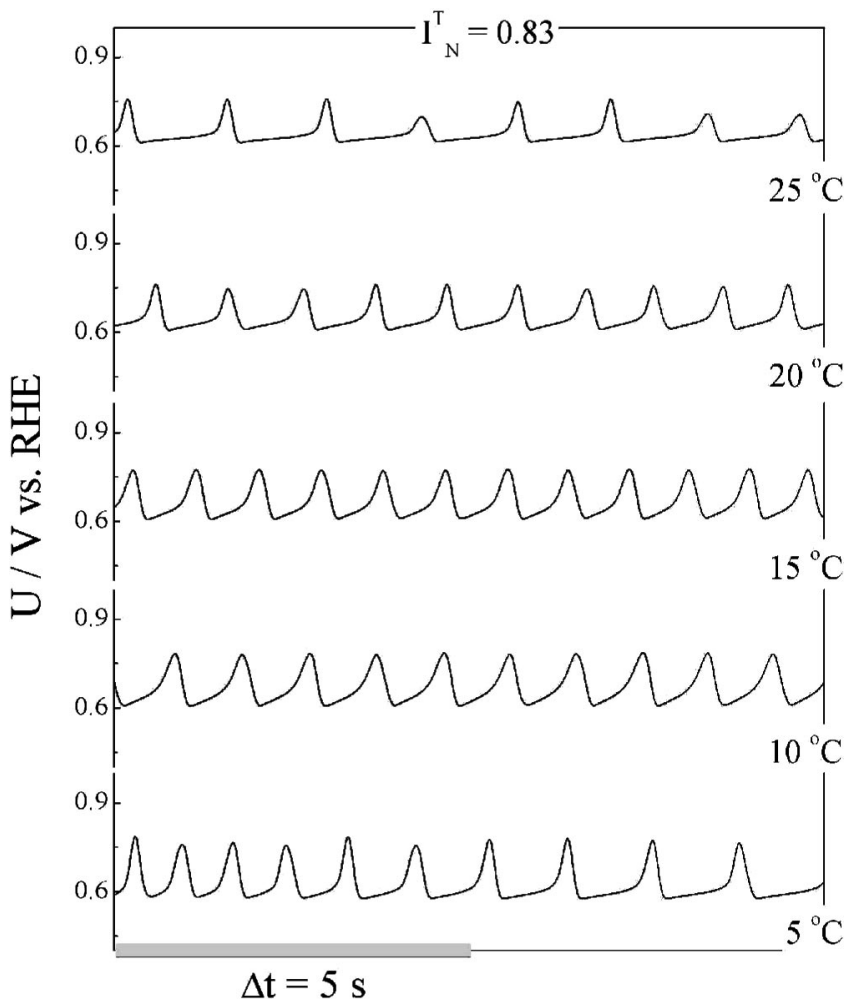

Figure 5. Potential time series at different temperatures and $I_{\mathrm{N}}^{T}=0.83$. Electrolyte: aqueous solution with $0.60 \mathrm{M} \mathrm{HCOOH}$ and $0.10 \mathrm{M} \mathrm{H}_{2} \mathrm{SO}_{4}$.

of the oscillations have also been reported for the hydrogen peroxide/thiosulfate/sulfite flow system. ${ }^{33}$ Specifically, a temperature increase from 25 to $33{ }^{\circ} \mathrm{C}$ accelerates the transition from low to high $\mathrm{pH}$ states, whereas the oscillation period and the transition from high to low $\mathrm{pH}$ values remain unaltered.

A summary of the results of the oscillation period and amplitude at different temperatures and for different applied currents is given in Figure 6. Period and amplitude were calculated for at least eight oscillations, and the uncertainty is given by the error bars. In both cases, the response is strongly dependent on the temperature and on the applied current. As shown in Figure 6a (note the logarithmic scale), the period increase accompanying the temperature increment is much more pronounced for low applied currents and becomes weaker at higher currents. The effect of temperature and applied current on the oscillation amplitude is more complicated. For the lowest current investigated, the amplitude increases linearly with temperature. This increase becomes less pronounced for higher currents, and finally a slight decrease is observed at the highest current value studied.

\section{Discussion}

1. Temperature (Over)Compensation of the Oscillation Period. In an oscillatory system, we may define the temperature coefficient in terms of the oscillation period $p$

$$
Q_{10}=\frac{p_{T}}{p_{T+10{ }^{\circ} \mathrm{C}}}
$$

or, the equivalent of eq 2 , for a generic temperature interval,

$$
q_{10}=\left(\frac{p_{T_{1}}}{p_{T_{2}}}\right)^{10 /\left(T_{2}-T_{1}\right)}
$$

The overall activation energy for an oscillatory system can be calculated in terms of the oscillation frequency $f(=1 / p),{ }^{42}$

$$
f=A \mathrm{e}^{-E_{\mathrm{a}} R T}
$$

or, similarly to eq 3 , in terms of the mean temperature coefficient $q_{10}$

In contrast to previous reports on chemical oscillators, ${ }^{32,33}$ oscillations in the electrocatalytic oxidation of formic acid on platinum occur over a wide temperature interval and were studied here in the range between 5 and $25{ }^{\circ} \mathrm{C}$. The mean temperature coefficient, as calculated from eq 6 , for different temperature intervals is given in Table 1. The last row in Table 1 was calculated over the whole temperature range with the activation energy (via eq 3), obtained from Arrhenius plots (ln $f$ vs $T^{-1}$ as in eq 7). In general, for a given temperature interval, $q_{10}$ tends to increase with the applied current. As mentioned in the Introduction, temperature overcompensation reflects a situation where a temperature increase results in a decrease in the reaction rate or, in the oscillatory state, in an increase of the oscillation period. As shown in Table 1, this is the situation for most cases, i.e., $q_{10}<1$. Temperature compensation of the oscillation period, or the near-independence of the oscillation period on temperature, is observed at high currents and at lower temperatures. For comparison, the temperature coefficient of

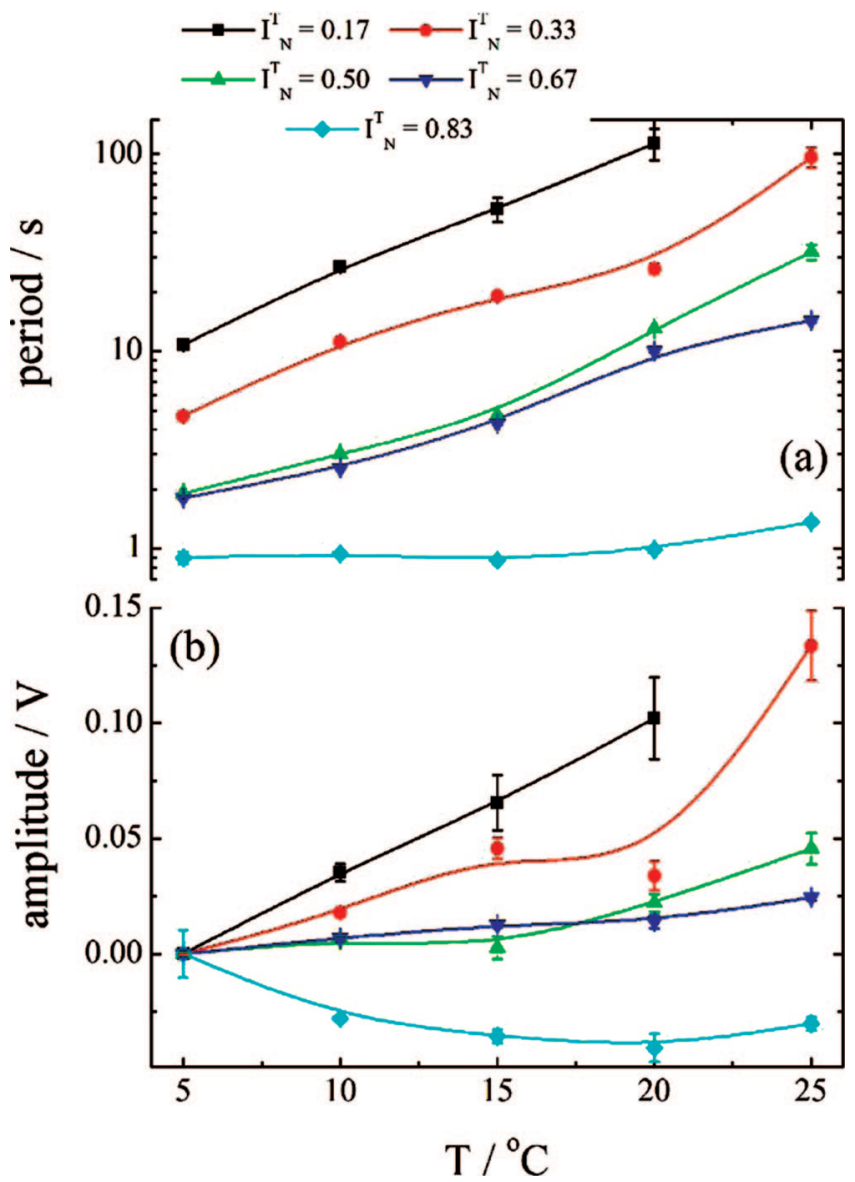

Figure 6. Oscillatory (a) period and (b) amplitude (value at $T$ minus value at $5{ }^{\circ} \mathrm{C}$ ) as a function of temperature for different normalized currents.

TABLE 1: $q_{10}$ Values Obtained for Different Temperature Intervals and Applied Currents

\begin{tabular}{lccccc}
\hline$\Delta T,{ }^{\circ} \mathrm{C}$ & $I_{\mathrm{N}}^{\mathrm{T}}=0.17$ & $I_{\mathrm{N}}^{\mathrm{T}}=0.33$ & $I_{\mathrm{N}}^{\mathrm{T}}=0.50$ & $I_{\mathrm{N}}^{\mathrm{T}}=0.67$ & $I_{\mathrm{N}}^{\mathrm{T}}=0.83$ \\
\hline $20-25$ & & 0.13 & 0.25 & 0.43 & 0.76 \\
$15-20$ & 0.22 & 0.53 & 0.13 & 0.18 & 0.78 \\
$10-15$ & 0.26 & 0.34 & 0.41 & 0.35 & 1.16 \\
$5-10$ & 0.16 & 0.18 & 0.40 & 0.50 & 0.92 \\
$5-25$ & 0.22 & 0.25 & 0.24 & 0.33 & 0.96
\end{tabular}


circadian oscillators usually ranges between 0.8 and 1.2. Temperature coefficients between 0.67 and 1.80 were obtained by Rábai and co-workers ${ }^{34}$ for the classic Bray reaction. Those experiments were carried out in batch, within a temperature interval of $10{ }^{\circ} \mathrm{C}$, and the $q_{10}$ values were found to be strongly dependent on the concentration of some reactants.

One question that arises when analyzing a temperaturecompensated chemical system is whether there is a real compensation mechanism or the system is simply insensitive to temperature. The results of the voltammetric sweep experiments shown in Figure 1 can help exclude the second possibility. As recently pointed out by Abruña and co-workers, ${ }^{43}$ the activation energy in processes like formic acid electrooxidation is a function of (a) potential, (b) the reaction pathway taken to reach that potential, and (c) the relative surface coverages of adsorbing anions, adsorbed organic residues such as $\mathrm{CO}_{\mathrm{ad}}$, and surface oxides. In that work, ${ }^{43}$ the authors studied in great detail the effect of temperature on the electrooxidation of methanol on platinum under several different experimental conditions and found a potential dependence similar to the one observed here in Figure 2. Following their interpretation, the highest value of the activation energy found here at around $840 \mathrm{mV}$ vs RHE is the one of interest, because the contributions of the surface processes mentioned above are minimized. Thus, the apparent activation energy of approximately $80 \mathrm{~kJ} \mathrm{~mol}^{-1}$ observed here can be taken as representative of the overall process of formic acid electrooxidation under nonoscillatory conditions. Therefore, the temperature coefficient around 1 , and consequently an activation energy around 0 , observed in the oscillatory state cannot simply be the result of a small dependence of the overall kinetics on temperature. A similar argument has been also used by Rábai and Hanazaki with regard to the temperature compensation observed in the homogeneous hydrogen peroxidethiosulfate-sulfide flow system. ${ }^{32}$

2. Oscillation Amplitude. The oscillation period and amplitude at different temperatures and for different applied currents were presented in Figure 6. The first noteworthy point is that both period and amplitude are strongly dependent on both temperature and applied current. It was observed that (a) at low applied currents a temperature increase results in an increase of the oscillation period and amplitude and (b) at high applied currents both period and amplitude are nearly independent of temperature. Different states were observed between these two limiting situations for different applied currents, and, as a general trend, it was seen that the higher the applied current the smaller the amplitude increase with temperature. At the same time, it was shown that overcompensation is favored at low applied currents and that the temperature coefficient increases for increasing applied currents.

In spite of the vast literature on temperature compensation of the oscillation period in biological systems, discussions concerning the dependence of the oscillation amplitude on temperature are less common and the results less conclusive. Indeed, the effect of temperature on the oscillatory amplitude in some temperature-compensated systems shows no clear tendency and depends on the clock properties at the biochemical level of the system. Lakin-Thomas et al. ${ }^{44}$ proposed a model for temperature-compensated systems in which the oscillation amplitude increases with temperature to keep the oscillation frequency constant. The "amplitude hypothesis" of LakinThomas et al. ${ }^{44}$ was used to explain the dynamics of phase resetting of the circadian oscillator in the fungus Neurospora crassa. Increase of the amplitude in the oscillatory production of the clock protein FRQ in the temperature-compensated circadian pacemaker of $N$. crassa was later observed in experiments ${ }^{45}$ and also captured in a simple model. ${ }^{3}$ From the purely chemical side, the increase of oscillation amplitude with temperature, as suggested by Lakin-Thomas et al., ${ }^{44}$ has been observed in the temperature-compensated hydrogen peroxideiodate ion chemical system (the Bray reaction) in batch. ${ }^{34}$ Pittendrigh et al. ${ }^{46}$ studied different aspects of the amplitude of Drosophila circadian pacemaker and observed that, unlike its period, the amplitude of the pacemaker is strongly temperature dependent. The results reported for Drosophila ${ }^{46}$ differ from those reported for Neurospora ${ }^{44}$ in terms of the temperature dependence of the oscillation amplitude: a temperature rise results in an amplitude decrease in the former case and in an amplitude increase in the latter. Moreover, the authors stressed the importance of understanding the effect of environmental constraints on the oscillation amplitude and suggest that the oscillation amplitude is one way in which circadian systems may feel seasonal cycles. ${ }^{46}$

The results obtained here point to an intricate interplay between temperature and applied current in the dynamics of our electrochemical oscillator. The current applied through the solid-liquid interface is a direct measure of the electron flow between the solid electrode and the species in solution. Therefore, the applied current is related to the departure from thermodynamic equilibrium and finds its analog in the mass flow rate in flow reactors. The influence of the relative position with respect to thermodynamic equilibrium on the way in which temperature affects the oscillation period and amplitude has not yet been addressed in depth. Using a theoretical model for a relaxation oscillator, Rössler ${ }^{47}$ speculated on the role of flow when designing a temperature-compensated homogenous chemical oscillator. In the proposed mechanism, he suggested that a near-zero flow would be sufficient for temperature compensation to be achieved, but there appears to be no experimental realization of such a chemical oscillator, and thus no verification of this prediction.

Studying the effect of temperature on the dynamics of some bromate oscillators catalyzed by cerium, Nagy et al. ${ }^{48}$ observed temperature coefficients in the range between 2.2 and 3.2 when the system was operated under batch conditions, whereas $q_{10}$ between 1.3 and 1.7 were observed in a continuous flow stirred tank reactor (CSTR). Although the authors recognized the important role of adding flow to a closed system to achieve temperature compensation, ${ }^{48}$ the impact of the flow rate on the oscillation amplitude was not presented. Studies of the hydrogen peroxide/thiosulfate/sulfite flow system $^{32}$ revealed that, in general, the oscillation amplitude decreases gradually with increasing temperature. In addition, the period was found to decrease with increasing flow rate. This system has been extensively studied ${ }^{49}$ and several different dynamic states observed. In some cases, the oscillation amplitude was found to increase for decreasing flow rate. However, no data concerning the influence of the flow rate, and thus the distance from equilibrium, on the oscillation amplitude for the temperaturecompensated case, were presented. Similar observations on the importance of the flow conditions on the temperature coefficient have been provided by Kovács and Rábai for $\mathrm{pH}$ oscillators. ${ }^{33}$ Numerical simulations show a decrease in the oscillation amplitude when the temperature is increased. Studying the effect of temperature on the oscillatory decomposition of hydrogen peroxide in an acidic aqueous solution in the presence of potassium iodate (Bray reaction), Kovács et al. ${ }^{34}$ observed in CSTR experiments that, for a certain flow rate, the oscillation 
amplitude increases for increasing temperature when temperature compensation is achieved.

We believe that the apparent variability in the effect of temperature on the oscillation amplitude, as reviewed above, may arise from the fact that the distance from thermodynamic equilibrium was not recognized as an important parameter. As presented here, e.g., Figure $6 b$, temperature may affect the oscillation amplitude in different ways depending on the distance from equilibrium. Whether the behavior reported here is universal is a question to be tested in other systems. The ease with which the distance from equilibrium can be controlled in reactions taking place at the solid/liquid interface, under either potentiostatic or galvanostatic conditions, makes electrochemical systems attractive analogs for studying temperature effects in biological systems.

3. Mechanistic Considerations. The electrocatalytic oxidation of $\mathrm{C} 1$ molecules is of interest from both the fundamental and applied points of view. In spite of the significant progress in understanding the mechanistic pathways of these important oxidation reactions, many fundamental aspects still remains obscure. Key questions such as the role of surface structure on activity, the effect of electrode material, etc., have been studied using carbon monoxide, formic acid and methanol as model molecules. ${ }^{37,50}$ It has been proposed that the electrooxidation of small organic molecules generally proceeds via a dual pathway mechanism ${ }^{51-53}$ in which two parallel reaction pathways occur simultaneously. A direct pathway occurs via formation of short lived active intermediates which, once formed, are promptly converted to the final product, carbon dioxide. In the indirect pathway, adsorbed carbon monoxide poisons the electrode surface, which is only freed at high overpotentials when $\mathrm{CO}_{\mathrm{ad}}$ is oxidized by adsorbed oxygenated species, $\left(\mathrm{H}_{x}\right) \mathrm{O}_{\mathrm{ads}}$, via a Langmuir-Hinshelwood $(\mathrm{LH})$ mechanism: ${ }^{54}$

$$
\mathrm{CO}_{\mathrm{ad}}+\left(\mathrm{H}_{x}\right) \mathrm{O}_{\mathrm{ad}} \rightarrow \mathrm{CO}_{2}+x \mathrm{H}^{+}+x \mathrm{e}^{-}+2 \mathrm{Pt}
$$

where $x$ can be 0,1 or 2 , according to the nature of the adsorbed oxygenated species. Step (r1) is also influenced by the competition between oxygenated species and adsorbing anions from the supporting electrolyte. ${ }^{37}$

Mainly due to the spectroscopic observation of adsorbed formate species, the dual pathway mechanism for formic acid electrooxidation has recently been revised. In contrast to previous statements, ${ }^{55-58}$ Behm and co-workers ${ }^{59-61}$ found that an adsorbed bridge-bonded formate species does not take part as a reaction intermediate in the direct pathway, but rather it acts as a spectator that blocks the catalyst surface in the, dominant, direct pathway. The authors propose a triple pathway mechanism which includes the direct pathway, ${ }^{61}$

$$
\mathrm{HCOOH}_{\mathrm{ad}} \stackrel{-2 \mathrm{H}^{+},-2 \mathrm{e}^{-}}{\longrightarrow} \mathrm{CO}_{2}
$$

the indirect pathway

$$
\mathrm{HCOOH}_{\mathrm{ad}} \stackrel{-\mathrm{H}_{2} \mathrm{O}}{\longrightarrow} \mathrm{CO}_{\mathrm{ad}} \stackrel{+\mathrm{H}_{2} \mathrm{O},-2 \mathrm{H}^{+},-2 \mathrm{e}^{-}}{=} \mathrm{CO}_{2} \quad(\mathrm{r} 3, \mathrm{r} 4)
$$

and the formate pathway

$$
\mathrm{HCOOH}_{\mathrm{ad}} \stackrel{-\mathrm{H}^{+},-\mathrm{e}^{-}}{\longrightarrow} \mathrm{HCOO}_{\mathrm{ad}}
$$

Oscillatory kinetics during the electrooxidation of formic acid on platinum has been observed many times ${ }^{62-71}$ and is known to result from the presence of a negative differential resistance in an N-shaped current potential curve. ${ }^{72}$ The phenomenon has been explained in terms of the interaction between positive and negative feedback loops acting on the electrode potential and can be rationalized in terms of the interaction of ${ }^{73}$ (a) surface poisoning along the indirect pathway, (b) cleaning of the surface by the reaction between adsorbed carbon monoxide and oxygenated species, and (c) feedback between the total surface coverage and the electrode potential. In terms of the oscillating adsorbed species, as part of the positive feedback loop on the electrode potential, the coverage of oxygenated species is in-phase with the potential oscillations. ${ }^{69}$ Additionally, the potential oscillations are in-phase with changes in the formate coverage and out-ofphase with the changes in carbon monoxide coverage. ${ }^{57}$

The key to understanding the mechanistic origin of the nonArrhenius behavior reported here is the effect of temperature on the different reaction steps that, when coupled, give rise to oscillations. Following the concept of antagonistic balance, ${ }^{14-16}$ the temperature (over)compensation observed here results from the interplay between the opposing effects of temperature on the positive feedback loop, which includes the steps of $\left(\mathrm{H}_{x}\right) \mathrm{O}$ adsorption, (bi)sulfate adsorption, $\mathrm{CO}_{\mathrm{ad}}$ oxidation by $\left(\mathrm{H}_{\mathrm{x}}\right) \mathrm{O}_{\mathrm{ad}}$ species, and formate adsorption and desorption/oxidation, and on the negative feedback loop composed of the (non-faradaic) step of formic acid dehydration to $\mathrm{CO}_{\mathrm{ad}}$. In all the time series reported here, the system was found to spend most of its time in the active, low potential state and only a short time in the passive, high potential state. Analyzing the time series in Figure 4 , it becomes evident that changing the temperature affects only the active to passive transition, whereas the steeper passive to active transition remains nearly unaffected by temperature. This is true for all applied currents studied. As shown in Figure 5, for higher currents the passive to active transition becomes slower, but still there is no discernible effect of temperature on the time spent in the passive state. This fact can be understood in terms of the proposed mechanism as being a consequence of the weak influence of temperature on the surface poisoning step (r3) or on the buildup of the carbon monoxide layer under the experimental conditions studied here, i.e., in the oscillatory regime and in the temperature range between 5 and $25^{\circ} \mathrm{C}$. As recently showed by Osawa and co-workers, ${ }^{57}$ the carbon monoxide coverage oscillates from $0.2-0.3$ to about $0.35-0.4$ monolayers during potential oscillations and under conditions similar to those employed here.

Analysis of temperature compensation phenomena for a chemical/metabolic oscillator often employs the sum of the activation energies of the several processes involved weighted by control coefficients. ${ }^{3}$ Thus, reliable values of the activation energy of each step are crucial for modeling the phenomena of temperature compensation and estimating the control coefficients. The determination of activation energies in coupled surface reactions is far from trivial. As already pointed out, the activation energy in complex processes such as the electrooxidation of formic acid depends on several parameters, including the electrode potential, the reaction pathway taken to reach that potential, the relative surface coverages of adsorbing anions, adsorbed organic residues such as $\mathrm{CO}_{\mathrm{ad}}$, and the presence of surface oxides. ${ }^{43}$ The changes in those surface coverages are certainly the major complication in evaluating the activation energies of the individual steps under oscillatory conditions.

To give an example, Osawa and co-workers ${ }^{57}$ observed that an increase in the applied current affects both the higher and lower limits of the carbon monoxide and formate coverages accompanying the potential oscillations. Specifically, the authors found that higher applied current results in a decrease in the 
mean coverage of carbon monoxide and a concomitant decrease in the mean coverage of formate species. Additionally, the complex surface chemistry in systems such as the electrooxidation of formic acid might bring about large configurational entropy effects, which in turn might result in nonstandard preexponential terms in the reaction rates. ${ }^{67}$ In this case, the temperature independence of the pre-exponential factor might break down and, as a consequence, some assumptions of the theory of temperature compensation ${ }^{3}$ would need revision before it can be applied to a network of coupled surface catalyzed reactions.

4. Extensions to Other Surface Reactions. The temperature (over)compensation observed here during the oscillatory electrooxidation of formic acid on platinum is, to the best of our knowledge, the first observation of such phenomena in a surface catalyzed reaction. We have also performed experiments aimed at investigating the influence of temperature on the oscillatory dynamics during methanol electrooxidation under conditions resembling those reported here. Although preliminary, these experiments reveal so far only conventional, Arrhenius, behavior, which suggests that, in spite of the mechanistic similarities of the oscillatory dynamics, the phenomena reported here seem to be specific to formic acid electrooxidation. Whether the temperature (over)compensation reported here is a unique phenomenon connected to some particular mechanistic steps of the electrooxidation of formic acid remains an open question. An important point in this context is the fact that such kinetic peculiarities arise only when the system is kept far from equilibrium.

5. Conclusions and Perspectives. Our experimental studies of the impact of temperature on the oscillatory electrooxidation of formic acid on a polycrystalline platinum surface and in acid media have revealed unique features. Apparent activation energies estimated under nonoscillatory conditions during slow voltammetric sweeps were found to range between 50 and 80 $\mathrm{kJ} \mathrm{mol}^{-1}$. In contrast, under oscillatory conditions, only nonArrhenius behavior was observed, with temperature overcompensation or compensation and a strong dependence of both period and amplitude on temperature and applied current. In particular, it was observed that (a) at low applied currents a temperature increase results in an increase of the oscillation period and amplitude and (b) at high applied currents both period and amplitude are nearly independent of temperature. The high, positive apparent activation energies obtained under voltammetric, nonoscillatory, conditions demonstrate that the non-Arrhenius behavior observed under oscillatory conditions results from the interplay among reaction steps rather than from a weak temperature dependence of the individual steps.

Given the few experimental data available on the intricate interaction between temperature and the distance from thermodynamic equilibrium and its effect on the dynamics of oscillatory systems, we believe the results reported here suggest the possibility of using electrocatalytic reactions such as the electrooxidation of formic acid on platinum based catalysts as chemical analogs of temperature-compensated biosystems. In particular, the effects of temperature and the distance from thermodynamic equilibrium on the oscillation period and amplitude reported here might contribute to answering the question "Is anything but the period at constant temperature regulated?", posed by Winfree. ${ }^{74}$

From the electrocatalytic perspective, the temperature (over)compensation observed here during the oscillatory electrooxidation of formic acid on platinum is the first observation of such phenomena in a surface catalyzed reaction. Further experiments using related systems will be performed to check whether the observed phenomena are universal or particular to the system studied here.

Finally, studying the effect of temperature on wave phenomena in an array of temperature-compensated electrochemical oscillators in a spatially extended configuration might provide insight into the interplay between spatial coupling and temperature in pattern forming systems. Studies of the solid/liquid interface would also benefit from the possibility of controling the type, intensity and locality of the spatial coupling. The unusual properties of the electrochemical oxidation of formic acid on platinum make this system a prime candidate for such investigations.

Acknowledgment. R.N. acknowledges Fundação de Amparo à Pesquisa do Estado de São Paulo (FAPESP) for scholarships (05/04667-3 and 06/04636-3). H.V. acknowledges (FAPESP) for financial support (04/04528-0). Discussions with Prof. J. C. Borges on some biochemical aspects of temperature compensation in living systems are gratefully acknowledged. I.R.E. was supported by the U.S. National Science Foundation (CHE0615507).

\section{References and Notes}

(1) Guerasimov, Y. A.; Dreving, V.; Eriomin, E.; Kiseliov, A.; Lebedev, V.; Panchekov, G.; Shliguin, A. Curso de Quimica Fisica, Tomo, II. Ed.; Mir: Morcu, 1971.

(2) Glasstone, S. Textbook of Physical Chemistry; Macmilllan and Co. Limited: London, 1962.

(3) Ruoff, P.; Loros, J. J.; Dunlap, J. C. Proc. Natl. Acad. Sci. U.S.A. 2005, 102, 17681-17686.

(4) Glass, L.; Mackey, M. C. From Clocks to Chaos: The Rhythms of Life; Princeton University Press: Princeton, NJ, 1988.

(5) Pittendrigh, C. S. Annu. Rev. Physiol. 1993, 55, 16-54.

(6) Iwasaki, K.; Liu, D. W.; Thomas, J. H. Proc. Natl. Acad. Sci. U.S.A. 1995, 92, 10317-10321.

(7) Ruoff, P.; Vinsjevik, M.; Rensing, L. Commun. Theor. Biol. 2000, 5, 361-382.

(8) Kyriacou, C. P.; Hall, J. C. Proc. Natl. Acad. Sci. U.S.A. 1980, 77, 6729-6733.

(9) Lloyd, D.; Edwards, S. W.; Fry, J. C. Proc. Natl. Acad. Sci. U.S.A. 1982, 79, 3785-3788.

(10) Ruoff, P.; Rensing, L. J. Therm. Biol. 2004, 29, 445-456.

(11) Mozurkewich, M.; Benson, S. W. J. Phys. Chem. 1984, 88, 64296435 .

(12) Mozurkewich, M.; Lamb, J. L.; Benson, S. W. J. Phys. Chem. 1984, $88,6435-6441$.

(13) Lamb, J. L.; Mozurkewich, M.; Benson, S. W. J. Phys. Chem. 1984, $88,6441-6448$.

(14) Hastings, J. W.; Sweeney, B. M. Proc. Natl. Acad. Sci. USA 1957, 43,804 .

(15) Ruoff, P. J. Interdiscipl. Cycle Res. 1992, 23, 92-99.

(16) Ruoff, P. Naturwissenschaften 1994, 81, 456-459.

(17) Huang, Z. J.; Curtin, K. D.; Rosbash, M. Science 1995, 267, 11691172.

(18) Somero, G.N.; Low, P. S. Am. Nat. 1977, 111, 527-538.

(19) Müller, S. C.; Hauser, M. J. B. Patterns and Waves in Chemistry and Biology. In Handbook of Biomimetics; Osaka, Y., Kai, S., Kakazu, Y., Kataoka, K., Sakai, K., Tanaka, J., Eds.; NTS Books: Tokyo, 2000; pp87. (20) Heathcote, H. L. Z. Phys. Chem. 1901, 37, 368-373.

(21) Lillie, R. S. Science 1918, 48, 51-60.

(22) Keizer, J.; Rock, P. A.; Lin, S. W. J. Am. Chem. Soc. 1979, 101, 5637-5649.

(23) Smolin, S.; Imbihl, R. J. Phys. Chem. 1996, 100, 19055-19058.

(24) Zaikin, A. N.; Zhabotinsky, A. M. Nature 1970, 225, 535.

(25) Epstein, I. R.; Pojman, J. A. An Introduction to Nonlinear Chemical Dynamics: Oscillations Waves, Patterns and Chaos; Oxford University Press: New York, 1998.

(26) Köros, E. Nature 1974, 251, 703-704.

(27) Ruoff, P. Physica D 1995, 84, 204-211.

(28) Blandamer, M. J.; Morris, S. H. J. Chem. Soc., Faraday Trans. 1975, 71, 2319-2330.

(29) Cervellati, R.; Furrow, S. D.; Pompeis, S. D. Int. J. Chem. Kinet. 2002, 34, 357-365.

(30) Cervellati, R.; Honer, K.; Furrow, S. D.; Mazzanti, F.; Costa, S. Helv. Chim. Acta 2004, 87, 133-155. 
(31) Kereszturi, K.; Szalai, I. Z. Phys. Chem. 2006, 220, 1071-1082.

(32) Rábai, G.; Hanazaki, I. Chem. Commun. 1999, 1965-1966.

(33) Kovács, K. M.; Rábai, G. Phys. Chem. Chem. Phys. 2002, 4, 52655269

(34) Kovács, K.; Hussami, L. L.; Rábai, G. J. Phys. Chem. A 2005, 109, 10302-10306.

(35) Tremiliosi-Filho, G.; Jerkiewicz, G.; Conway, B. E. Langmuir 1992, $8,658-667$.

(36) Varela, H.; Krischer, K. J. Phys. Chem. B 2002, 106, 12258-12266.

(37) Markovic, N.; Ross, P. N. Surf. Sci. Rep. 2002, 45, 117-229.

(38) Varela, H. Spatiotemporal Pattern Formation during Electrochemical Oxidation of Hydrogen on Platinum. Ph.D. Thesis, Freie Universität Berlin, 2003.

(39) Varela, H.; Beta, C.; Bonnefont, A.; Krischer, K. Phys. Rev. Lett. 2005, 94, 174104-1-174104-4.

(40) Varela, H.; Krischer, K. Catal. Today 2001, 70, 411-425.

(41) Plenge, F.; Varela, H.; Lübke, M.; Krischer, K. Z. Phys. Chem. 2003, 217, 365-381.

(42) Zhang, J.; Datta, R. J. Electrochem. Soc. 2002, 149, A1423-1431.

(43) Cohen, J. L.; Volpe, D. J.; Abruña, H. D Phys. Chem. Chem. Phys. 2007, 9, 49-77.

(44) Lakin-Thomas, P. L.; Brody, S.; Coté, G. C. J. Biol. Rhythm. 1991, 6, 281-297

(45) Liu, Y.; Merrow, M.; Loros, J. J.; Dunlap, J. C Science 1998, 281, $825-829$.

(46) Pittendrigh, C. S.; Kyner, W. T.; Takamura, T. J. Biol. Rhythm. 1991, 6, 299-313.

(47) Rössler, O. E. Proc. San Diego Bimed. Symp., San Diego 1975, 99,105

(48) Nagy, G.; Körös, E.; Oftedal, N.; Tjelflaat, K.; Ruoff, P. Chem. Phys. Lett. 1996, 250, 255-260.

(49) Rábai, G.; Hanazaki, I. J. Phys. Chem. A 1999, 103, 7268-7273.

(50) Jarvi, T. D.; Stuve, E. Fundamental aspects of vacuum and electrocatalytic reactions of methanol and formic acid on platinum surfaces. In Electrocatalysis; Lipkowski, J., Ross, P. N., Eds.; Wiley-VCH: New York, Chapter 3, p75-154, 1998.

(51) Capon, A.; Parsons, R. J. Electroanal. Chem. 1973, 44, 1-7.

(52) Capon, A.; Parsons, R. J. Electroanal. Chem. 1973, 44, 239-254.

(53) Capon, A.; Parsons, R. J. Electroanal. Chem. 1973, 45, 205-231.

(54) Laidler, K. J. Chemical Kinetics, 3rd ed.; Harper \& Row Publishers: New York, 1987.

(55) Miki, A.; Osawa, M. Chem. Commun. 2002, 1500-1501.
(56) Samjeske, G.; Osawa, M. Angew. Chem. Int. Ed. 2005, 44, 56945698

(57) Samjeske, G.; Miki, A.; Ye, S.; Yamakata, A.; Mukouyama, Y; Okamoto, H.; Osawa, M. J. Phys. Chem. B 2005, 109, 23509-23516.

(58) Samjeske, G.; Miki, A.; Ye, S.; Osawa, M. J. Phys. Chem. B 2006, $110,16559-16566$.

(59) Chen, Y. X.; Heinen, M.; Jusys, Z.; Behm, R. J. Angew. Chem. Int. Ed. 2006, 45, 981-985.

(60) Chen, Y. X.; Heinen, M.; Jusys, Z.; Osawa, M.; Behm, R. J. J. Phys. Chem. B 2006, 110, 9534-9544.

(61) Chen, Y. X.; Heinen, M.; Jusys, Z.; Behm, R. J. Langmuir 2006, $22,10399-10408$

(62) Wojtowicz, J.; Marincic, N.; Conway, B. E. J. Chem. Phys. 1968, 48, 4333-4345.

(63) Anastasijevic, N. A.; Baltruschat, H.; Heitbaum, J. J. Electroanal. Chem. 1989, 272, 89-100.

(64) Schell, M.; Albahadily, F. N.; Safar, J.; Xu, Y. J. Phys. Chem. 1989, 93, 4806-4810.

(65) Raspel, F.; Nichols, R. J.; Kolb, D. M. J. Electroanal. Chem. 1990, 286, 279-283.

(66) Albahadily, F. N.; Schell, M. J. Electroanal. Chem. 1991, 308, 151173.

(67) Markovic, N.; Ross, P. N. J. Phys. Chem. 1993, 97, 9771-9778.

(68) Strasser, P.; Lübke, M.; Raspel, F.; Eiswirth, M.; Ertl, G. J. Chem. Phys. 1997, 107, 979-990.

(69) Strasser, P.; Eiswirth, M.; Ertl, G. J. Chem. Phys. 1997, 107, 9911003

(70) Honda, Y.; Song, M. B.; Ito, M. Chem. Phys. Lett. 1997, 273, 141146.

(71) Schmidt, T. J.; Grgur, B. N.; Markovic, N. M.; Ross, P. N. J. Electroanal. Chem. 2001, 500, 36-43.

(72) Strasser, P.; Eiswirth, M.; Koper, M. T. M. J. Electroanal. Chem. 1999, 478, 66.

(73) Krischer, K.; Varela, H. Oscillations and Other Dynamic Instabilities. In Handbook of Fuel Cells: Fundamentals, Technology, Applications; Vielstich, W., Gasteiger, H. A., Lamm, A., Eds.; John Wiley \& Sons: Chichester, 2003; Vol. 2, pp 679-701.

(74) Winfree, A. T. The Geometry of Biological Time, 2nd ed.; Springer: New York, 2000.

JP801361J 